\title{
Cognitive control of movement via the cerebellar-recipient thalamus
}

\section{Vincent Prevosto* and Marc A. Sommer}

Department of Biomedical Engineering, The Center for Cognitive Neuroscience, The Duke Institute for Brain Sciences, Duke University, Durham, NC, USA

\section{Edited by:}

Yuri B. Saalmann, University of

Wisconsin - Madison, USA

Reviewed by:

Richard P. Dum, University of

Pittsburgh, USA

Eiji Hoshi, Tokyo Metropolitan

Institute of Medical Science, Japan

*Correspondence:

Vincent Prevosto, Center for

Cognitive Neuroscience, Duke

University, Box 90999, Durham, NC

27708, USA

e-mail:vincent.prevosto@duke.edu
The cognitive control of behavior was long considered to be centralized in cerebral cortex. More recently, subcortical structures such as cerebellum and basal ganglia have been implicated in cognitive functions as well. The fact that subcortico-cortical circuits for the control of movement involve the thalamus prompts the notion that activity in movement-related thalamus may also reflect elements of cognitive behavior. Yet this hypothesis has rarely been investigated. Using the pathways linking cerebellum to cerebral cortex via the thalamus as a template, we review evidence that the motor thalamus, together with movement-related central thalamus have the requisite connectivity and activity to mediate cognitive aspects of movement control.

Keywords: motor thalamus, central thalamus, thalamus, cognition, cerebellum, timing, executive control, language

\section{INTRODUCTION}

The majority of our knowledge of the primate thalamus at the systems level is based on the study of circuits for sensation (e.g., retinogeniculostriate pathway). Questions of how thalamic circuits contribute to movement and cognition are largely unanswered. The more complex the behavior, the more that motor and cognitive processes will need to interact with each other. Imagine, as a brief example, the actions and calculations that are intertwined as a driver merges into highway traffic. The degree to which motor and cognitive processes may co-occur is constrained by environmental factors (Knoblich and Flach, 2001; Pulvermüller and Fadiga, 2010; Filimon et al., 2013), but it is well accepted that motor and cognitive systems must be able to share information and run simultaneously (Cisek and Kalaska, 2010; Koziol et al., 2012). Here we review evidence for cognitive processes in movement-related thalamus, with special emphasis on cognitive functions that are particularly developed in primates, as opposed to more common functions such as associative learning that are found in all vertebrates, or even arthropods (Giurfa, 2013).

Motor thalamus is classically delineated according to cerebellar and basal ganglia projection zones. This review will primarily focus on the two juxtaposed thalamic regions that receive inputs from so-called motor and non-motor domains of the dentate nucleus, the output node of the lateral cerebellum. The first region corresponds to typical cerebellar territories of the motor thalamus (Figure 1A, left, and violet in Figure 1B), which are essentially found posteriorly to basal ganglia territories, in the ventral lateral complex (VL) of the thalamic nuclei (VLps and VLc subdivisions as well as nucleus $\mathrm{X}$ ) and the oral division of the ventral posterolateral nucleus (VPLo). Those thalamic nuclei in turn project to cortical motor areas [primary motor cortex (M1), premotor cortex (PM) and the supplementary motor area (SMA)]. Additionally, projections from nucleus $\mathrm{X}$ and caudal regions of VLc also target the pre-SMA and frontal and parietal associative cortices (Wiesendanger and Wiesendanger, 1985a,b; Middleton and Strick, 2001; Morel et al., 2005; Prevosto et al., 2010). The second thalamic region considered in this review is composed of the central thalamus (Figure 1A, right, and green in Figure 1B). This region contains the rostral intralaminar complex [mainly the central lateral nucleus (CL) and, for cerebellar territories, to a lesser extent the paracentral nucleus $(\mathrm{Pcn})]$ together with paralaminar regions of the mediodorsal nucleus (MD) and $\mathrm{VL}$ (Schlag-Rey and Schlag, 1989; Groenewegen and Berendse, 1994). The posterior intralaminar system (centre médian and parafascicular nuclei), heavily interconnected with basal ganglia, will not be discussed here. The central thalamus targets association cortices as well as motor cortices, with a gradient of projections (Rouiller et al., 1999; Morel et al., 2005; Prevosto et al., 2010). Most cortical regions that receive cerebellar inputs are recipients of thalamic inputs from these two contiguous thalamic regions, with different weights. As mentioned above, motor thalamus predominantly targets motor cortical regions. In contrast, central thalamus has widespread access to both associative and motor cortex.

\section{CONTEXTUAL MODULATION OF ACTIVITY IN THALAMUS AND CEREBELLUM}

The influence of cognitive functions on the neuronal activity of motor thalamus is far from established. It is well known, however, that only a subset of neurons in the motor thalamus is concerned solely with basic motor parameters. Many of the neurons contribute, instead, to more elaborate features of movement planning and execution. This functional distinction is in agreement with findings that both cerebellum and basal ganglia are implicated in higher level functions that expand and complement their role in movement (Middleton and Strick, 1994; Aglioti, 1997; Haber and Calzavara, 2009). Similarly, motor thalamus, as classically defined by its subcortical inputs, has long been known to project to cortical regions well beyond motor and PM (e.g., Kievit and Kuypers, 

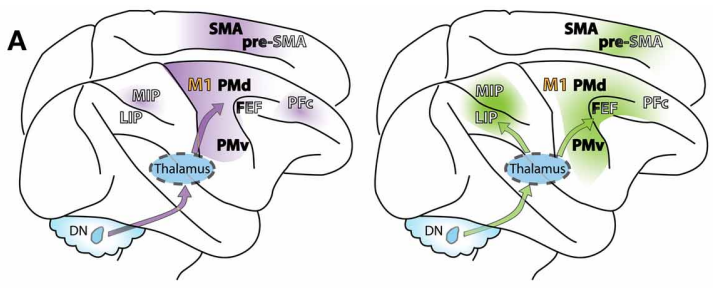

B
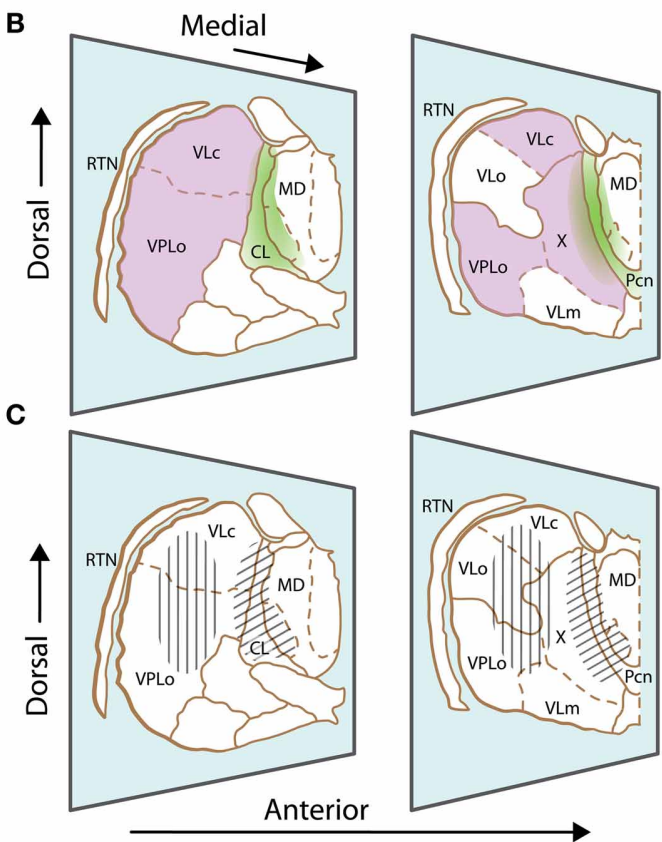

FIGURE 1 | The motor thalamus and ascending cerebellar inputs to cerebral cortex. (A) Lateral view of the rhesus monkey brain with hemispheres separated to expose mesial wall on top. Arrows schematically represent cerebello-cortical pathways relayed via cerebellar territories in lateral (left, violet) and central thalamus (right, green). Gradients of color on cortex indicate relative strength of inputs. Left: PFc, MIP are set apart, as inputs to such non-motor cortices are relayed via medial and dorsal regions of the classic motor thalamus, topographically distinct from regions relaying inputs to motor cortices. Names of cortical areas are color-coded as follows: Primary motor cortex, orange; non-primary motor fields, black; association cortices, white (non-exhaustive presentation). FEF and Pre-SMA have dual color-coding, as they receive inputs from "non-motor" cerebellar domains, but display some non-primary motor features as well. (B) Two representative sections of the thalamus of Macaca mulatta, viewed from a lateral anterior perspective (thalamic nuclei delineated according to (Olszewski, 1952)). Only relevant nuclei are labeled. Reticular thalamic nucleus (RTN) is not part of the motor thalamus but mediates cortical inhibitory control of thalamic activity. Cerebellar domains in lateral and central thalamus are presented in violet and green, respectively. The diffuse borders of central thalamus encompass the rostral intralaminar group (central lateral nucleus, $\mathrm{CL}$, and the paracentral nucleus, $\mathrm{Pcn}$ ) and paralaminar regions of the VL complex and MD. (C) Same sections as (B), but illustrating effector-related functional, as opposed to hodological, compartments. Hatching shows the rough somatotopic locations of regions related to arm (vertical) and eye (diagonal) movements. Other abbreviations: DN, dentate nucleus; MIP, medial intraparietal area; LIP, lateral intraparietal area; M1, primary motor cortex; PMd, dorsal premotor cortex; PMv, ventral premotor cortex; FEF, frontal eye fields; PFc, prefrontal cortex; SMA, supplementary motor area; pre-SMA, pre-supplementary motor area; VLo, ventrolateral nucleus pars oralis; VPLo, ventroposterolateral nucleus pars oralis; MD, mediodorsal nucleus; VLc, ventrolateral nucleus pars caudalis, $X$, nucleus $X$ of the thalamus.
1977). Conversely, top down cortical control that mediates cognitive signals from the prefrontal cortex (Brunia, 1999) may also influence movement-related activity in motor thalamus. This latter control is thought to be an important factor in volitional and selective gating of ascending inputs (Nadeau, 2008). As will be discussed below, volitional and context-dependent modulation of activity are hallmarks of cognitive influence on movement-related processing in the thalamus.

A classic demonstration that motor-related activity in thalamus is not always tightly associated with specific movement parameters came from oculomotor research (Schlag and SchlagRey, 1984; Schlag-Rey and Schlag, 1984). In their seminal papers, Schlag-Rey and Schlag introduced a bold proposal, namely the central controller hypothesis, which proposed that eye-movement related activity in the central thalamus specifies the timing of particular actions. The activity modulations in central thalamic regions are highly sensitive to context, and their specific sets of projections have been shown to mediate aspects of cognitive processing such as working memory (van der Werf et al., 2002). Indeed, if the context in which a movement is made influences neuronal activity during motor preparation and execution, this information must be stored and effectively accessed; in other words, working memory properties are needed (for more details on primate working memory circuits, see Constantinidis and Procyk, 2004).

Similar findings of contextually modulated activity, as well as selective modulation of preparatory activity for volitional movements, have been observed in the cerebellar dentate nucleus (Grimm and Rushmer, 1974; Mushiake and Strick, 1993; Ashmore and Sommer, 2013; Prevosto et al., 2013), the source of cerebello-thalamic projections from the lateral cerebellum. These results support the concept that some cerebello-thalamocortical pathways may be involved in higher order aspects of motor control and behavior. This suggestion raises two related issues. First, considering the potential involvement of cerebellocortical circuits in cognitive functions that underlie complex behavior (Diamond, 2000; Koziol et al., 2012), what evidence exists that thalamus mediates the relevant activities? Then, for those activities that motor thalamus conveys, does the thalamus have an active, participatory role, or does it primarily act as a relay?

\section{CEREBELLO-THALAMO-CORTICAL CIRCUITS HAVE THE REQUISITE CONNECTIVITY FOR COGNITIVE INVOLVEMENT}

Although it gradually emerged that motor regions of the thalamus project to a wide array of cortical targets outside agranular (motor) cortex (Kievit and Kuypers, 1977; Wiesendanger and Wiesendanger, 1985b; Schmahmann and Pandya, 1990; Shook et al., 1991), relating those pathways to their subcortical sources has proven difficult. Indeed, beyond the confusion arising from diverse nomenclatures (Percheron et al., 1996), a structural definition of the motor thalamus has always been complicated by the fact that ascending axonal arborizations cover regions that straddle multiple cytoarchitectonically-defined nuclei (Kalil, 1981; Percheron et al., 1996; Mason et al., 2000). Conversely, thalamocortical projections originate from longitudinal regions that cross over nuclei borders (Kievit and Kuypers, 1977; Percheron et 
al., 1996). Therefore, the input-output arrangement of thalamic pathways is one of tremendous complexity.

Only with the advent of transneuronal tracers has it been possible to map with precision the reciprocal, polysynaptic pathways between cerebellar output nuclei and associative regions outside of the motor cortices (Lynch et al., 1994; Clower et al., 2001; Kelly and Strick, 2003; Ramnani, 2006; Strick et al., 2009; Hashimoto et al., 2010; Prevosto et al., 2010; Lu et al., 2012) (Figure 1A). It appears that the majority of cortical areas, notably prefrontal, medial frontal, and posterior parietal regions providing inputs to the cerebellum (via the pontine nuclei), in turn receive cerebellothalamo-cortical inputs (Strick et al., 2009; Ramnani, 2012). Curiously, a number of cortical regions thought to be crucial for cognition, such as the rostral temporal lobe and the ventrolateral prefrontal cortex, do not participate in these closed loops. This fact suggests a commonality between cortical areas that communicate with the cerebellum: it appears that they all contribute to the planning, control, or monitoring of movement.

The diversity of lateral cerebellar output channels, however, raises the question of their organization. Formerly, the loops that traverse lateral cerebellum through VL (Figures 1A,B) (Asanuma et al., 1983; Stein and Glickstein, 1992) were seen primarily as pathways for posterior parietal areas to gain access to PM (Thach, 1987; Stein and Glickstein, 1992), in agreement with known VL contributions to movement planning (Strick, 1976). However, the modern understanding that cerebro-cerebellar connections are largely reciprocal, and consequently target a variety of cortical areas outside the motor cortices, forces a re-evaluation of the ways in which lateral cerebellum, and its thalamic targets, may contribute to behavior. As explained above, lateral cerebellar ascending projections may be divided largely into two streams, one relayed via motor thalamus, the other via central thalamus. It is tempting to attribute the origin of each stream to motor and non-motor domains of the dentate nucleus respectively, with corresponding motor and cognitive functions. However, while cerebellar output channels are essentially segregated from each other, many cortical areas receive inputs from both central and more lateral thalamic regions (Figures 1A,B), making it difficult to separate both streams. In the two following sections, we will attempt to illuminate how the two thalamic regions differ in their contributions to the cognitive control of movement.

\section{COGNITIVE-RELATED INPUTS TO CENTRAL THALAMUS}

It is notable that identified cerebellar projections to central thalamic regions (formerly "non-specific" thalamus; Sasaki et al., 1979; Kalil, 1981; Asanuma et al., 1983; Sultan et al., 2012) were first considered to be potential output pathways for cerebellar cognitive signals (Leiner et al., 1986). This hypothesis assumed that inputs relayed through the central thalamus would constitute a separate, "non-specific" pathway that would exert a general influence through widespread thalamocortical projections. This view is compatible with the fact that central thalamus targets not only association cortices but also PM, SMA, and pre-SMA with considerable divergence (Figure 1A; Morel et al., 2005). This projection system, however, has been shown to be much more specific than previously conceived (van der Werf et al., 2002) and can influence selective regions, in addition to having a general impact on cortical activation levels. Specific influences carried via central thalamus, such as the modulation of preparatory activity mentioned above, would likely have different temporal dynamics than motor-related signal carried by the motor thalamus. Functional distinction between central and motor thalamus is less obvious at the transition zone in medial and dorsal parts of VL. Indeed, the fact that cerebellar inputs to prefrontal cortex seem to be relayed via caudal VLc and nucleus X (Middleton and Strick, 2001) argue for an involvement of motor thalamic regions in higher-level functions (see below). Accordingly, it has been proposed that cerebellar-recipient neurons of the caudal regions of central thalamus may be considered part of a functional continuum with more lateral "motor" cerebellar territories (Percheron et al., 1996). It has also been suggested that the mediodorsal (MD) thalamic nucleus, the main source of thalamic inputs to prefrontal cortex (Giguere and Goldman-Rakic, 1988; Ray and Price, 1993), may convey cerebellar signals (Sasaki et al., 1979; Tian and Lynch, 1997). If so, cerebellar inputs to MD would be expected to be found alongside motor signals from the superior colliculus (SC), which are relayed by the lateral $\mathrm{MD}$ to the frontal eye fields (FEF) (Sommer, 2003). Paralaminar regions of $\mathrm{MD}$, however, are dominantly innervated by basal ganglia inputs, and cerebellar projections there are limited (Stanton, 1980; Kalil, 1981; Percheron et al., 1996; Mason et al., 2000; Erickson et al., 2004). It is thus likely that the majority of ascending cerebellar projections to frontal associative cortex is transmitted either via central thalamus or more lateral cerebellar territories (formerly "classical" motor thalamus).

Eye-movement related circuits show the limitations in distinguishing these pathways purely based on connectivity. The oculomotor thalamus largely overlaps with central thalamus (Schlag-Rey and Schlag, 1989; Tanaka and Kunimatsu, 2011) (Figures 1B,C, right) and targets both the lateral intraparietal area (LIP) and the FEF (Kievit and Kuypers, 1977; Huerta et al., 1986; Prevosto et al., 2010) (Figure 1A), two prominent nodes in the cortical circuits for the selection and control of eye movement. Both of these cortical regions receive inputs from the same caudal dentate region (Lynch et al., 1994; Prevosto et al., 2010). However, in comparison to LIP, dentate inputs to FEF may also be relayed via more lateral (paralaminar) thalamic regions (Okuda, 1994).

How this functional ensemble may contribute to higher level function is starting to be understood. For instance, central thalamus is known to contribute to working memory via its action on forebrain arousal (Mair et al., 2011). This action has often been related to the ascending reticular activation system, which notably provides intralaminar nuclei with profuse cholinergic inputs (Groenewegen and Berendse, 1994). Central thalamus, however, has the requisite connectivity to mediate subcortical influence on selective cortical circuits. Recent results showing that intact cerebello-thalamo-cerebral pathways are crucial for the normal functioning of working memory (Law et al., 2011) are compatible with this view.

Recent data implicate the lateral cerebellum in verbal working memory, but also point out contributions to spatial processing, timing, and executive functions (Leiner et al., 1989, 1993; Chen and Desmond, 2005; Strick et al., 2009; Schmahmann, 2010; Bellebaum et al., 2012; Ramnani, 2012; Stoodley, 2012). The exact 
involvement of central thalamus in these functions is not yet clear, although there is evidence that it contributes to timing, in addition to working memory. Saccade-related neurons in central thalamus have been shown to display early activity that is particularly associated with the timing of self-initiated eye movements (Tanaka, 2007a; Tanaka and Kunimatsu, 2011). Complementary saccade-related activity patterns have been found in central thalamus that could signal the timing for acquisition and processing of reafferent information following saccades (Schlag-Rey and Schlag, 1984). Although neuronal activity related to self-initiated eye movements has been observed in basal ganglia, the thalamic neurons related to the timing of proactive movements were found predominantly in cerebellar territory (Tanaka, 2007a), in agreement with the putative involvement of the dentate nucleus in the initiation of volitional movements (Shibasaki et al., 1986; Ashmore and Sommer, 2013) (Figure 2). Thus, central thalamus appears well suited to transmit anticipatory activity related to volitional, self-timed movements from the lateral cerebellum to connected cortical areas (Maimon and Assad, 2006; Fried et al., 2011).

\section{COGNITIVE-RELATED INPUTS TO VL/VPLO THALAMUS}

While encouraging, the above conclusions were based largely on oculomotor studies. An important issue is the extent to which those findings generalize to skeletomotor movements, which are associated with thalamic activity in the VL and VPLo nuclei (Figures 1B,C, left).

Recordings in the cerebellar dentate nucleus (a dominant contributor to VL, see above) have described a population of neurons with long-lead activity (Grimm and Rushmer, 1974; Strick, 1983). Correspondingly, among arm-movement VL units, most cerebellar-recipient neurons increase their discharge before movement initiation, sometimes before the first change in electromyographic potential (Strick, 1976; Anderson and Turner, 1991). While the cerebellum is known for its role in the timing

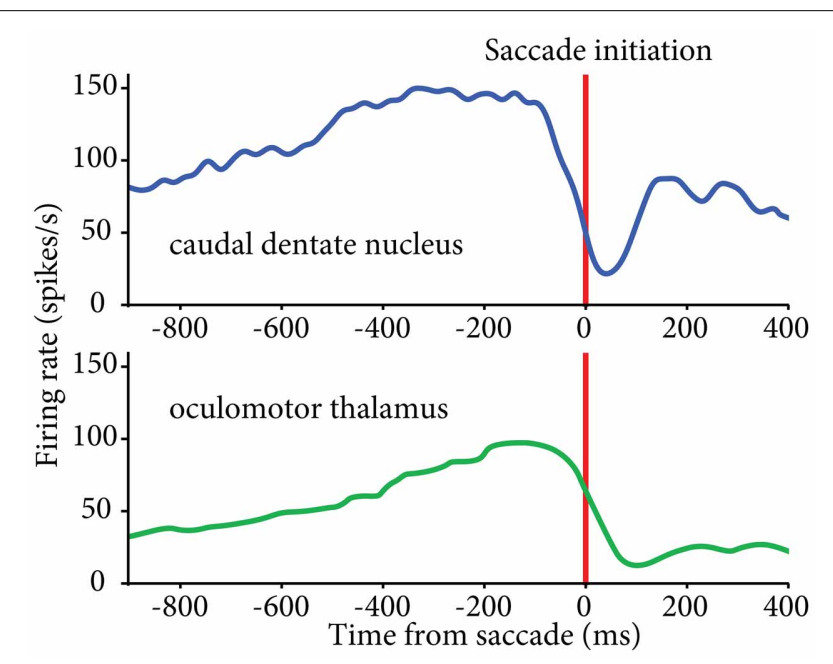

FIGURE 2 | Similar patterns of activity found in caudal dentate nucleus (top) and oculomotor thalamus (bottom) for self-initiated eye movements. Activity aligned to the initiation of self-timed saccades. Oculomotor thalamus data reproduced from Tanaka (2007a); dentate nucleus data from our lab (see Ashmore and Sommer, 2013). of movement (Salman, 2002), the involvement of cerebellarrecipient motor thalamus in the timing of volitional armmovement is less clear. Notably, arm-movement neurons in cerebellar-recipient thalamus are commonly found to be more responsive to visually cued movement than to spontaneous or memory-based movement (van Donkelaar et al., 1999). Similar contextual modulation has been described in neurons from the "motor" domain of the dentate that target ventral premotor cortex (Mushiake and Strick, 1993) as well as in the medial intraparietal area (Colby and Duhamel, 1996), a region of the posterior parietal cortex that receives dentate inputs via motor thalamus (Prevosto et al., 2010). However, comparable effects have been described across effectors and brain structures, as exemplified by greater response to visually-guided than to spontaneous arm movements (van Donkelaar et al., 1999, 2000) and saccades (Mano et al., 1996) in thalamus and cerebellum, respectively. Thus, context dependency of neuronal activity may reflect a widespread influence found across functional divisions of the thalamus. Notably, a growing body of imaging and clinical studies (Ide and Li, 2011; Peterburs et al., 2011; van der Salm et al., 2013) indicate that cerebellar territories of the motor thalamus provide a critical contribution to executive control functions of the frontal lobe. This contribution could rely on motor thalamic inputs to non-primary motor regions and (less densely) to associative cortical regions, or central thalamic inputs to the same regions, or both (Figure 1A).

More speculative is the potential role of motor thalamus in coordinating cognitive and motor aspects of language production. Results from stimulation studies found that language deficits can be induced at the same thalamic location as motor effects related to language production (Johnson and Ojemann, 2000). This intriguing finding is in agreement with the demonstration that ventral premotor cortex contains neurons specifically activated during vocalization (Coudé et al., 2011), in a region that receives dense projections from cerebellar-recipient thalamus (Matelli et al., 1989). This ventral premotor region is considered homologous to the motor portion of Broca's area in humans (Binkofski and Buccino, 2004) and is part of a dual cerebellocortical system supporting verbal working memory (Chen and Desmond, 2005).

\section{THE THALAMUS AS A SITE FOR MOTOR/COGNITIVE INTERACTIONS}

If the motor and central thalamic neurons are involved in higherorder functions, the question remains whether their activities are driven mainly by their ascending inputs (i.e., whether thalamic neurons are just relays), or if these inputs, coupled with descending cortical modulation, results in thalamus-specific information processing.

Evidence from the well-characterized circuit from the SC to FEF via MD thalamus mentioned above (Sommer and Wurtz, 2004a,b) offers a template for a relay function: SC-receiving MD relay neurons essentially behave as a high-pass filtered version of SC inputs (Sommer and Wurtz, 2004a). This is fitting with the role of this circuit as a corollary-discharge pathway, i.e., carrying copies of a motor command.

Cerebellar-receiving thalamic neurons, which are not necessarily part of a corollary-discharge pathway, may behave differently. 
It is known that the activity of motor thalamic neurons is shaped by cortical inputs (Guillery, 1995). This is evidenced, for example, by the high baseline firing rate of pallidal-recipient thalamic neurons, which likely results from a dual modulatory cortical control, one direct and excitatory, the other indirect and inhibitory (Selemon and Goldman-Rakic, 1988; Anderson and Turner, 1991; Guillery, 1995; Band and van Boxtel, 1999). Similarly, the activity of eye position thalamic neurons reflects properties of both brainstem inputs (separate horizontal/vertical channels; delays compatible with ascending inputs) and cortical inputs (hysteresis; long lead activity) (Schlag and Schlag-Rey, 1984; Tanaka, $2007 b$ ), in agreement with the view of intralaminar nuclei as a site of convergence of subcortical and cortical inputs (Kemp and Powell, 1971). It is thus conceivable that cortical inputs modulate cerebellar-recipient neurons' activity at least as strongly as their primary drive.

Another type of thalamic-specific interaction potentially occurs through converging ascending inputs from multiple subcortical sources. Demonstrated convergence patterns of this type, such as between dentate and interpositus nucleus projections (Shinoda et al., 1985), or cerebellar and basal ganglia projections (Sakai et al., 2002), have been studied only within pathways contributing to motor cortical areas, and are essentially inconclusive for the question of motor-cognitive interaction in the thalamus. However, the thalamus also has been shown to convey cerebellar inputs to striatum that derive from both motor and non-motor regions of the dentate nucleus (Kemp and Powell, 1971; Hoshi et al., 2005). Interestingly, the central thalamus seems to be the main relay for this pathway (Ichinohe et al., 2000; Hoshi et al., 2005). It is conceivable that, reciprocally, basal ganglia inputs to cerebellar thalamic regions contribute to both motor and non-motor circuits.

The two preceding types of interactions (subcortico-cortical and subcortico-subcortical) point to a dominant role of central thalamus in mediating cognitive aspects of movement control. Another aspect of thalamic connectivity suggest a third way by which both motor a central thalamic regions could actively contribute to cognitive control of movement. There is evidence that single thalamic regions provide inputs to functionally separate cortical areas, such as motor and associative cortices (Wannier et al., 1992). This divergence seems to represent a final sorting of signals that arise from selective regions of the dentate nucleus. Data from separate studies suggest that dentate regions where output channels overlap could target distributed cerebral cortical regions. Such is the case for caudal dentate projections to the FEF and LIP (Lynch et al., 1994; Prevosto et al., 2010), for dentate projections to the anterior intraparietal area, PMv, and M1 (Clower et al., 2005), and possibly for ventral dentate to the

\section{REFERENCES}

Aglioti, S. (1997). The role of the thalamus and basal ganglia in human cognition. J. Neurolinguist. 10, 255-265. doi: 10.1016/S0911-6044(97)00020-1

Akkal, D., Dum, R., and Strick, P. (2007). Supplementary motor area and presupplementary motor area: targets of basal ganglia and cerebellar output. J. Neurosci. 27, 10659-10673. doi: 10.1523/JNEUROSCI.3134-07.2007 Anderson, M. E., and Turner, R. S. (1991). Activity of neurons in cerebellar-receiving and pallidal-receiving areas of the thalamus of the behaving

medial intraparietal area and the pre-SMA (Wiesendanger and Wiesendanger, 1985a; Akkal et al., 2007; Prevosto et al., 2010). While this organization does not validate a specific role for the thalamus in motor-cognitive interaction, it indicates that there may be a unique and overlooked role of motor thalamus in conveying such signals to separate regions. How those dentate projections interact in the thalamus with top-down cortical control and ascending inputs from other subcortical regions is not known, although it can be construed that this arrangement would likely contribute to behavioral flexibility.

\section{CONCLUSIONS}

Concordant results from a variety of studies indicate that movement-related thalamus takes part in circuits for higher-level control of behavior. A prior, parsimonious viewpoint was that distinct subcortico-thalamo-cortical pathways were likely to mediate separate functions (e.g., Sommer, 2003). Recent findings seem to paint a more nuanced picture.

First, each pathway consists of sub-streams, both in the thalamus and in the subcortical networks leading to it. These sub-streams may play differing roles in movement, or perhaps have differing contributions according to behavioral context. Accordingly, cerebellar-receiving thalamic neurons are possibly involved in both straightforward visuomotor control and higher level modulations of that control, such as the initiation of selftimed movements (Figure 2). The degree to which these conclusions hold across effectors, however, is still unclear. Second, the discovery of reciprocal, disynaptic connections between the cerebellum and the basal ganglia (Bostan and Strick, 2010) imply direct communications between these two principal pathways to cerebral cortex. The fact the central thalamus is posited as the main relay for cerebellar inputs to striatum underlines its relevance for high-level behavior in association with "core" motor thalamus.

Hence the overall conclusion from clinical, physiological, and anatomical studies is that the thalamus appears suited to relay, or perhaps even to mediate, the influence of cognitive processes on motor processes. Because in mammals, and particularly in primates, most behaviors comprise a cognitive component, it is not surprising to find prevalent cognitive modulation of motor circuits. The surprise comes perhaps from the fact that circuits beyond the cerebral cortex, including nuclei of the motor and central thalamus, seem to be so critical for cognitive-motor interactions.

\section{ACKNOWLEDGMENTS}

Supported by NIH R01 EY017592 and R21 EY022788 to Marc A. Sommer.

monkey. J. Neurophysiol. 66, 879-893.

Asanuma, C., Thach, W., and Jones, E. (1983). Anatomical evidence for segregated focal groupings of efferent cells and their terminal ramifications in the cerebellothalamic pathway of the monkey. Brain Res. 286, 267-297.
Ashmore, R., and Sommer, M. (2013). Delay activity of saccade-related neurons in the caudal dentate nucleus of the macaque cerebellum. J. Neurophysiol. 109, 2129-2144. doi: 10.1152/jn.00906.2011

Band, G., and van Boxtel, G. (1999). Inhibitory motor control in stop paradigms: 
review and reinterpretation of neural mechanisms. Acta Psychol. 101, 179-211. doi: 10.1016/S0001-6918(99)00005-0

Bellebaum, C., Daum, I., and Suchan, B. (2012). Mechanisms of cerebellar contributions to cognition in humans. Wiley Interdiscip. Rev. Cogn. Sci. 3, 171-184. doi: 10.1002/wcs.1161

Binkofski, F., and Buccino, G. (2004). Motor functions of the Broca's region. Brain Lang. $\quad 89, \quad 362-369$. doi: 10.1016/S0093-934X(03)00358-4

Bostan, A., and Strick, P. (2010). The cerebellum and basal ganglia are interconnected. Neuropsychol. Rev. 20, 261-270. doi: 10.1007/s11065010-9143-9

Brunia, C. (1999). Neural aspects of anticipatory behavior. Acta Psychol. 101, 213-242. doi: 10.1016/S00016918(99)00006-2

Chen, S., and Desmond, J. (2005). Temporal dynamics of cerebro-cerebellar network recruitment during a cognitive task. Neuropsychologia 43, 1227-1237. doi: $\quad 10.1016 /$ j.neuropsychologia. 2004.12.015

Cisek, P., and Kalaska, J. (2010). Neural mechanisms for interacting with a world full of action choices. Annu. Rev. Neurosci. 33, 269-298. doi: 10.1146/annurev.neuro.051508. 135409

Clower, D., Dum, R., and Strick, P. (2005). Basal ganglia and cerebellar inputs to 'AIP'. Cereb. Cortex 15, 913-920. doi: 10.1093/cercor/bhh190

Clower, D., West, R., Lynch, J., and Strick, P. (2001). The inferior parietal lobule is the target of output from the superior colliculus, hippocampus, and cerebellum. J. Neurosci. 21, 6283-6291.

Colby, C. L., and Duhamel, J. R. (1996). Spatial representations for action in parietal cortex. Brain Res. Cogn. Brain Res. 5, 105-115. doi: 10.1016/S0926-6410(96)00046-8

Constantinidis, C., and Procyk, E. (2004). The primate working memory networks. Cogn. Affect. Behav. Neurosci. 4, 444-465. doi: 10.3758/CABN.4.4.444

Coudé, G., Ferrari, P., Rodà, F., Maranesi, M., Borelli, E., Veroni, V., et al. (2011). Neurons controlling voluntary vocalization in the macaque ventral premotor cortex. PLoS ONE 6:e26822. doi: 10.1371/journal.pone.0026822

Diamond, A. (2000). Close interrelation of motor development and cognitive development and of the cerebellum and prefrontal cortex. Child
Dev. 71, 44-56. doi: 10.1111/14678624.00117

Erickson, S., Melchitzky, D., and Lewis, D. (2004). Subcortical afferents to the lateral mediodorsal thalamus in cynomolgus monkeys. Neuroscience 129, 675-690. doi: 10.1016/j.neuroscience.2004.08.016

Filimon, F., Philiastides, M., Nelson, J., Kloosterman, N., and Heekeren, H. (2013). How embodied is perceptual decision making? Evidence for separate processing of perceptual and motor decisions. J. Neurosci. 33, 2121-2136. doi: 10.1523/JNEUROSCI.2334-12.2013

Fried, I., Mukamel, R., and Kreiman, G. (2011). Internally generated preactivation of single neurons in human medial frontal cortex predicts volition. Neuron 69, 548-562. doi: 10.1016/j.neuron.2010.11.045

Giguere, M., and Goldman-Rakic, P. (1988). Mediodorsal nucleus: areal, laminar, and tangential distribution of afferents and efferents in the frontal lobe of rhesus monkeys. J. Comp. Neurol. 277, 195-213. doi: $10.1002 /$ cne. 902770204

Giurfa, M. (2013). Cognition with few neurons: higher-order learning in insects. Trends Neurosci. $\quad 36, \quad 285-294 . \quad$ doi: 10.1016/j.tins.2012.12.011

Grimm, R., and Rushmer, D. (1974). The activity of dentate neurons during an arm movement sequence. Brain Res. 71, 309-326. doi: 10.1016/0006-8993(74)90974-3

Groenewegen, H., and Berendse, H. (1994). The specificity of the 'nonspecific' midline and intralaminar thalamic nuclei. Trends Neurosci. 17, 52-57. doi: 10.1016/0166-2236(94)90074-4

Guillery, R. (1995). Anatomical evidence concerning the role of the thalamus in corticocortical communication: a brief review. J. Anat. 187(Pt 3), 583-592

Haber, S., and Calzavara, R. (2009). The cortico-basal ganglia integrative network: the role of the thalamus. Brain Res. Bull. 78, 69-74. doi: 10.1016/j.brainresbull.2008.09.013

Hashimoto, M., Takahara, D., Hirata, Y., Inoue, K.-I., Miyachi, S., Nambu, A., et al. (2010). Motor and nonmotor projections from the cerebellum to rostrocaudally distinct sectors of the dorsal premotor cortex in macaques. Eur. J. Neurosci. 31, 1402-1413. doi: 10.1111/j.14609568.2010.07151.x

Hoshi, E., Tremblay, L., Féger, J., Carras, P., and Strick, P. (2005). The cerebellum communicates with the basal ganglia. Nat. Neurosci. 8, 1491-1493. doi: 10.1038/nn1544
Huerta, M., Krubitzer, L., and Kaas, J. (1986). Frontal eye field as defined by intracortical microstimulation in squirrel monkeys, owl monkeys, and macaque monkeys: I. Subcortical connections. J. Comp. Neurol. 253, 415-439. doi: 10.1002/cne.902530402

Ichinohe, N., Mori, F., and Shoumura, K. (2000). A di-synaptic projection from the lateral cerebellar nucleus to the laterodorsal part of the striatum via the central lateral nucleus of the thalamus in the rat. Brain Res. 880, 191-197. doi: 10.1016/S00068993(00)02744-X

Ide, J., and Li, C.-S. R. (2011). A cerebellar thalamic cortical circuit for error-related cognitive control. Neuroimage 54, 455-464. doi: $\quad 10.1016 /$ j.neuroimage. 2010 07.042

Johnson, M., and Ojemann, G. (2000) The role of the human thalamus in language and memory: evidence from electrophysiologica studies. Brain Cogn. 42, 218-230. doi: 10.1006/brcg.1999.1101

Kalil, K. (1981). Projections of the cerebellar and dorsal column nuclei upon the thalamus of the rhesus monkey. J. Comp. Neurol. 195, 25-50. doi: 10.1002/cne.901950105

Kelly, R., and Strick, P. (2003) Cerebellar loops with motor cortex and prefrontal cortex of a nonhuman primate. J. Neurosci. 23 , 8432-8444.

Kemp, J., and Powell, T. (1971). The connexions of the striatum and globus pallidus: synthesis and speculation. Philos. Trans. R. Soc. Long. B Biol. Sci. 262, 441-457. doi: 10.1098/rstb.1971.0106

Kievit, J., and Kuypers, H. (1977). Organization of the thalamocortical connexions to the frontal lobe in the rhesus monkey. Exp. Brain Res. 29, 299-322. doi: 10.1007/BF00236173

Knoblich, G., and Flach, R. (2001). Predicting the effects of actions interactions of perception and action. Psychol. Sci. 12, 467-472. doi: 10.1111/1467-9280.00387

Koziol, L., Budding, D., and Chidekel, D. (2012). From movement to thought: executive function, embodied cognition, and the cerebellum. Cerebellum 11, 505-525. doi: 10.1007/s12311-011-0321-y

Law, N., Bouffet, E., Laughlin, S., Laperriere, N., Brière, M.-E., Strother, D., et al. (2011). Cerebellothalamo-cerebral connections in pediatric brain tumor patients: impact on working memory. Neuroimage 56, 2238-2248. doi: 10.1016/j.neuroimage.2011.03.065
Leiner, H., Leiner, A., and Dow, R. (1986). Does the cerebellum contribute to mental skills? Behav. Neurosci. 100, 443-454. doi: 10.1037/0735-7044.100.4.443

Leiner, H., Leiner, A., and Dow, R (1989). Reappraising the cerebellum: what does the hindbrain contribute to the forebrain? Behav. Neurosci. 103, 998-1008. doi: 10.1037/0735-7044.103.5.998

Leiner, H., Leiner, A., and Dow, R. (1993). Cognitive and language functions of the human cerebellum. Trends Neurosci. 16, 444-447. doi: 10.1016/0166-2236(93)90072-T

Lu, X., Miyachi, S., and Takada, M. (2012). Anatomical evidence for the involvement of medial cerebellar output from the interpositus nuclei in cognitive functions. Proc. Natl. Acad. Sci. U.S.A. 109, 18980-18984. doi: 10.1073/pnas.1211168109

Lynch, J., Hoover, J., and Strick, P. (1994). Input to the primate frontal eye field from the substantia nigra, superior colliculus, and dentate nucleus demonstrated by transneuronal transport. Exp. Brain Res. 100, 181-186. doi: 10.1007/BF00227293

Maimon, G., and Assad, J. (2006). A cognitive signal for the proactive timing of action in macaque LIP. Nat. Neurosci. 9, 948-955. doi: 10.1038/nn1716

Mair, R., Onos, K., and Hembrook, J. (2011). Cognitive activation by central thalamic stimulation: the yerkes-dodson law revisited. Dose Response 9, 313-331. doi: 10.2203/dose-response.10-017.Mair Mano, N., Ito, Y., and Shibutani, H. (1996). Context dependent discharge characteristics of saccaderelated Purkinje cells in the cerebellar hemispheres of the monkey. Prog. Brain Res. 112, 423-430. doi: 10.1016/S0079-6123(08)63347-6

Mason, A., Ilinsky, I. A., Maldonado, S., and Kultas-Ilinsky, K. (2000). Thalamic terminal fields of individual axons from the ventral part of the dentate nucleus of the cerebellum in Macaca mulatta. J. Comp. Neurol. 421, 412-428. doi: 10.1002/ (SICI)1096-9861(20000605)421:3< 412::AID-CNE9>3.3.CO;2-Q

Matelli, M., Luppino, G., Fogassi, L., and Rizzolatti, G. (1989). Thalamic input to inferior area 6 and area 4 in the macaque monkey. J. Comp. Neurol. 280, 468-488. doi: 10.1002/cne.902800311

Middleton, F., and Strick, P. (1994). Anatomical evidence for cerebellar and basal ganglia involvement in higher cognitive function. Science 266, 458-461. doi: 10.1126/science.7939688 
Middleton, F., and Strick, P. (2001). Cerebellar projections to the prefrontal cortex of the primate. J. Neurosci. 21, 700-712.

Morel, A., Liu, J., Wannier, T., Jeanmonod, D., and Rouiller, E. (2005). Divergence and convergence of thalamocortical projections to premotor and supplementary motor cortex: a multiple tracing study in the macaque monkey. Eur. J. Neurosci. 21, 1007-1029. doi: 10.1111/j.1460-9568.2005.03921.x

Mushiake, H., and Strick, P. (1993). Preferential activity of dentate neurons during limb movements guided by vision. J. Neurophysiol. 70, 2660-2664.

Nadeau, S. (2008). The thalamus and working memory. J. Int. Neuropsychol. Soc. 14, 900-901. doi: $10.1017 / S 1355617708081149$

Okuda, B. (1994). Cerebello-thalamocerebral projection from the dentate nucleus onto the frontal eye field in the cat. Acta Physiol. Scand. 151, 1-6. doi: 10.1111/j.17481716.1994.tb09715.x

Olszewski, J. (1952). The Thalamus of the Macaca mulatta: An Atlas for Use With the Stereotaxic Instrument. Basel: Karger.

Percheron, G., François, C., Talbi, B., Yelnik, J., and Fénelon, G. (1996). The primate motor thalamus. Brain Res. Rev. 22, 93-181. doi: 10.1016/0165-0173(96)00003-3

Peterburs, J., Pergola, G., Koch, B., Schwarz, M., Hoffmann, K.-P., Daum, I., et al. (2011). Altered error processing following vascular thalamic damage: evidence from an antisaccade task. PLoS ONE 6:e21517. doi: 10.1371/journal.pone.0021517

Prevosto, V., Graf, W., and Ugolini, G. (2010). Cerebellar inputs to intraparietal cortex areas LIP and MIP: functional frameworks for adaptive control of eye movements, reaching, and arm/eye/head movement coordination. Cereb. Cortex 20, 214-228. doi: 10.1093/cercor/bhp091

Prevosto, V., Raghavan, R. T., and Sommer, M. A. (2013). "Evidence for involvement of lateral cerebellum in executive control and performance monitoring," in Proceedings of the Society for Neuroscience (San Diego, CA).

Pulvermüller, F., and Fadiga, L. (2010). Active perception: sensorimotor circuits as a cortical basis for language. Nat. Rev. Neurosci. 11, 351-360. doi: 10.1038/nrn2811

Ramnani, N. (2006). The primate cortico-cerebellar system: anatomy and function. Nat. Rev. Neurosci. 7, 511-522. doi: 10.1038/nrn1953
Ramnani, N. (2012). Frontal lobe and posterior parietal contributions to the cortico-cerebellar system. Cerebellum 11, 366-383. doi: 10.1007/s12311-011-0272-3

Ray, J., and Price, J. (1993). The organization of projections from the mediodorsal nucleus of the thalamus to orbital and medial prefrontal cortex in macaque monkeys. J. Comp. Neurol. 337, 1-31. doi: 10.1002/cne.903370102

Rouiller, E., Tanne, J., Moret, V., and Boussaoud, D. (1999). Origin of thalamic inputs to the primary, premotor, and supplementary motor cortical areas and to area 46 in macaque monkeys: a multiple retrograde tracing study. J. Comp. Neurol. 409, 131-152. doi: 10.1002/(SICI) 1096-9 861(19990621)409:1<131::AID-CN E10>3.0.CO;2-A

Sakai, S., Inase, M., and Tanji, J. (2002). The relationship between MI and SMA afferents and cerebellar and pallidal efferents in the macaque monkey. Somatosens. Mot. Res. 19, 139-148. doi: 10.1080/08990220220131533

Salman, M. S. (2002). Topical review: the cerebellum: it's about time! but timing is not everything-new insights into the role of the cerebellum in timing motor and cognitive tasks. J. Child Neurol. 17, 1-9. doi: 10.1177/088307380201700101

Sasaki, K., Jinnai, K., Gemba, H., Hashimoto, S., and Mizuno, N. (1979). Projection of the cerebellar dentate nucleus onto the frontal association cortex in monkeys. Exp. Brain Res. 37, 193-198. doi: 10.1007/BF01474266

Schlag, J., and Schlag-Rey, M. (1984). Visuomotor functions of central thalamus in monkey. II. Unit activity related to visual events, targeting, and fixation. J. Neurophysiol. 51, 1175-1195.

Schlag-Rey, M., and Schlag, J. (1984). Visuomotor functions of central thalamus in monkey. I. Unit activity related to spontaneous eye movements. J. Neurophysiol. 51, 1149-1174.

Schlag-Rey, M., and Schlag, J. (1989). The central thalamus. Rev. Oculomot. Res. 3, 361-390.

Schmahmann, J. (2010). The role of the cerebellum in cognition and emotion: personal reflections since 1982 on the dysmetria of thought hypothesis, and its historical evolution from theory to therapy. Neuropsychol. Rev. 20, 236-260. doi: 10.1007/s11065-010-9142-x

Schmahmann, J., and Pandya, D. (1990). Anatomical investigation of projections from thalamus to posterior parietal cortex in the rhesus monkey: a WGA-HRP and fluorescent tracer study. J. Comp. Neurol. 295, 299-326. doi: 10.1002/cne. 902950212

Selemon, L., and Goldman-Rakic, P. (1988). Common cortical and subcortical targets of the dorsolateral prefrontal and posterior parietal cortices in the rhesus monkey: evidence for a distributed neural network subserving spatially guided behavior. J. Neurosci. 8, 4049-4068.

Shibasaki, H., Barrett, G., Neshige, R., Hirata, I., and Tomoda, H. (1986). Volitional movement is not preceded by cortical slow negativity in cerebellar dentate lesion in man. Brain Res. 368, 361-365. doi: 10.1016/0006-8993(86)90582-2

Shinoda, Y., Kano, M., and Futami, T. (1985). Synaptic organization of the cerebello-thalamo-cerebral pathway in the cat. I. Projection of individual cerebellar nuclei to single pyramidal tract neurons in areas 4 and 6. Neurosci. Res. 2, 133-156. doi 10.1016/0168-0102(85)90009-4

Shook, B., Schlag-Rey, M., and Schlag, J. (1991). Primate supplementary eye field. II. Comparative aspects of connections with the thalamus, corpus striatum, and related forebrain nuclei. J. Comp. Neurol. 307, 562-583. doi: 10.1002/cne.903070405

Sommer, M. (2003). The role of the thalamus in motor control. Curr. Opin. Neurobiol. 13, 663-670. doi: 10.1016/j.conb.2003.10.014

Sommer, M., and Wurtz, R. (2004a). What the brain stem tells the frontal cortex. I. Oculomotor signals sent from superior colliculus to frontal eye field via mediodorsal thalamus. J. Neurophysiol. 91, 1381-1402. doi: 10.1152/jn.00738.2003

Sommer, M., and Wurtz, R. (2004b). What the brain stem tells the frontal cortex. II. Role of the SC-MDFEF pathway in corollary discharge. J. Neurophysiol. 91, 1403-1423. doi: 10.1152/jn.00740.2003

Stanton, G. (1980). Topographical organization of ascending cerebellar projections from the dentate and interposed nuclei in Macaca mulatta: an anterograde degeneration study. J. Comp. Neurol. 190, 699-731. doi: 10.1002/cne.901900406

Stein, J., and Glickstein, M. (1992). Role of the cerebellum in visual guidance of movement. Physiol. Rev. 72, 967-1017.

Stoodley, C. (2012). The cerebellum and cognition: evidence from functional imaging studies.
Cerebellum 11, 352-365. doi: 10.1007/s12311-011-0260-7

Strick, P. (1976). Activity of ventrolateral thalamic neurons during arm movement. J. Neurophysiol. 39, 1032-1044.

Strick, P. (1983). The influence of motor preparation on the response of cerebellar neurons to limb displacements. J. Neurosci. 3, 2007-2020.

Strick, P., Dum, R., and Fiez, J. (2009). Cerebellum and nonmotor function. Annu. Rev. Neurosci. 32, 413-434. doi: 10.1146/annurev. neuro.31.060407.125606

Sultan, F., Augath, M., Hamodeh, S., Murayama, Y., Oeltermann, A., Rauch, A., et al. (2012). Unravelling cerebellar pathways with high temporal precision targeting motor and extensive sensory and parietal networks. Nat. Commun. 3, 924. doi: 10.1038/ncomms 1912

Tanaka, M. (2007a). Cognitive signals in the primate motor thalamus predict saccade timing. J. Neurosci. 27, 12109-12118. doi: 10.1523/JNEUROSCI.1873-07.2007

Tanaka, M. (2007b). Spatiotemporal properties of eye position signals in the primate central thalamus. Cereb. cortex 17, 1504-1515. doi: 10.1093/cercor/bhl061

Tanaka, M., and Kunimatsu, J. (2011). Contribution of the central thalamus to the generation of volitional saccades. Eur. J. Neurosci. 33, 2046-2057. doi: 10.1111/j.14609568.2011.07699.x

Thach, W. T. (1987). Cerebellar inputs to motor cortex. Ciba Found. Symp. 132, 201-230. doi: 10.1002/9780470513545.ch13

Tian, and Lynch, J. C. (1997). Subcortical input to the smooth and saccadic eye movement subregions of the frontal eye field in Cebus monkey. J. Neurosci. 17, 9233-9247. van der Salm, S., van der Meer, J., Nederveen, A., Veltman, D., van Rootselaar, A.-F., and Tijssen, M. (2013). Functional MRI study of response inhibition in Myoclonus Dystonia. Exp. Neurol. 247, 623-629. doi: 10.1016/j.expneurol.2013.02.017

van der Werf, Y., Witter, M., and Groenewegen, H. (2002). The intralaminar and midline nuclei of the thalamus. Anatomical and functional evidence for participation in processes of arousal and awareness. Brain Res. Rev. 39, 107-140. doi: 10.1016/S0165-0173(02)00181-9

van Donkelaar, P., Stein, J., Passingham, R., and Miall, R. (1999). Neuronal activity in the primate motor thalamus during visually triggered 
and internally generated limb movements. J. Neurophysiol. 82, 934-945.

van Donkelaar, P., Stein, J., Passingham, R., and Miall, R. (2000). Temporary inactivation in the primate motor thalamus during visually triggered and internally generated limb movements. J. Neurophysiol. 83, 2780-2790.

Wannier, T., Kakei, S., and Shinoda, Y. (1992). Two modes of cerebellar input to the parietal cortex in the cat. Exp. Brain Res. 90, 241-252. doi: 10.1007/BF00227235
Wiesendanger, R., and Wiesendanger, M. (1985a). Cerebello-cortical linkage in the monkey as revealed by transcellular labeling with the lectin wheat germ agglutinin conjugated to the marker horseradish peroxidase. Exp. Brain Res. 59, 105-117. doi: 10.1007/BF00237671

Wiesendanger, R., and Wiesendanger, M. (1985b). The thalamic connections with medial area 6 (supplementary motor cortex) in the monkey (Macaca fascicularis). Exp. Brain Res. 59, 91-104. doi: 10.1007/BF00237670
Conflict of Interest Statement: The authors declare that the research was conducted in the absence of any commercial or financial relationships that could be construed as a potential conflict of interest.

Received: 29 April 2013; accepted: 09 September 2013; published online: 01 October 2013.

Citation: Prevosto $V$ and Sommer MA (2013) Cognitive control of movement via the cerebellar-recipient thalamus. Front. Syst. Neurosci. 7:56. doi: 10.3389/ fnsys.2013.00056
This article was submitted to the journal Frontiers in Systems Neuroscience.

Copyright (c) 2013 Prevosto and Sommer. This is an open-access article distributed under the terms of the Creative Commons Attribution License (CC BY). The use, distribution or reproduction in other forums is permitted, provided the original author(s) or licensor are credited and that the original publication in this journal is cited, in accordance with accepted academic practice. No use, distribution or reproduction is permitted which does not comply with these terms. 